\title{
Influence Factors and Microstructures of Machined Surface in Electrical Discharge Machining for $\mathrm{SiC}_{\mathrm{w}} / 2024$ Aluminium Matrix Composite
}

\author{
Dazhen Wang ${ }^{1,}$,, Peifeng Feng ${ }^{1, b}$ \\ ${ }^{1}$ College of Mechanical and Energy Engineering of Jimei University, Xiamen 361021, China \\ aemail:dazhenwang@163.com.cn, bemail:pffeng@163.com.cn
}

\begin{abstract}
Keywords: $\mathrm{SiC}_{\mathrm{w}} / 2024$; aluminium matrix composites; electrical discharge machining (EDM); microstructure.

Abstract. The microstructures of machined surface of the specimens in electrical discharge machining(EDM) for $\mathrm{SiC}_{\mathrm{w}} / 2024$ aluminium matrix composites were measured with scanning electron microscope(SEM) and Talysurf-6 roughometer, and the energy spectrums of them were analyzed using EDAX spectrometer. The results show that the microstructures of machined surface are the "birds nest" shape complex structures, and mainly by the formation of pits, flange convexes, bonding of aluminium droplets again, cracks and $\mathrm{SiC}_{\mathrm{w}}$ whisker breakages. Moreover, deformation and failure modes of $\mathrm{SiC}_{\mathrm{w}}$ whiskers have three kinds of fusing and breaking off, pulling out directly, bonding again. With the increase of pulse width, processing speed and roughness values of the machined surface will increase, too. However, with the increase of pulse interval, processing speed will rapidly decrease, but roughness values of machined surface will maintain the stability.
\end{abstract}

\section{Introduction}

$\mathrm{SiC}_{\mathrm{w}} / 2024$ aluminum matrix composites have high specific strength, high specific stiffness, fatigue resistance, and low thermal expansion coefficient etc. They are widely used in aerospace, advanced weapon system, electronic industry, advanced cars and competitive sports[1].

However, due to the high stiffness and high-wearing feature of the reinforcements $\mathrm{SiC}_{\mathrm{w}}$ in composite, the carbide tools are only executed to cut the composite, which has low volume fraction, and fine size. However, for the composites with high volume fraction and big size, the cutting tools as carbide tools are quickly worn and to be failure in machining[2-4]. Despite of employing tool with high stiffness as polycrystalline diamond tool(PCD), it would still be difficult to have a satisfied results for the tool long life and the quality of machined surface. Therefore, it is important and necessary to research to machine the $\mathrm{SiC}_{\mathrm{w}}$ reinforced aluminium composite in non-traditional machining[5-7].

The electrical discharge machining $(E D M)$ is a remarkable technology, which has a great variety of merits[8]. Globally, the existing literatures only study the initial relationship between the group of parameters as the electrode, the electrode materials, the electric current and the width of discharging impulse etc and another group of parameters as the material removal rate, the wear rate of electrode and the roughness value of machined surface etc[9-11]. In this paper, $\mathrm{SiC}_{\mathrm{w}} / 2024$ aluminum matrix composites would be employed as the experimental materials for carrying out the tests of wire electrical discharge machining. Moreover, it is deeply researched that the microstructures of machined surface and the influences of electric parameters to processing speed and the quality of machined surface. These studies could facilitate the applied range of high-performance aluminium matrix composites and reduce the costs applied.

\section{Experimental materials and methods}

\section{Experimental materials}

The experimental materials applied are $\mathrm{SiC}_{\mathrm{w}} / 2024$ aluminum matrix composites, the diameter of whisker is $0.1 \sim 1 \mu \mathrm{m}$, length $10 \sim 80 \mu \mathrm{m}$. Their physical and mechanical performance has been listed in table 1 . 
Table 1 Physical and mechanical properties of the specimens

\begin{tabular}{cccccc}
\hline $\begin{array}{c}\text { Experimental } \\
\text { materials }\end{array}$ & $\begin{array}{c}\text { Volume fraction of } \\
\text { Reinforcements } \\
\varphi / \%\end{array}$ & $\begin{array}{c}\text { Density } \\
\rho / \text { g.cm }^{-3}\end{array}$ & $\begin{array}{c}\text { Tensile } \\
\text { Strength } \\
\sigma / \mathrm{MP}_{\mathrm{a}}\end{array}$ & $\begin{array}{c}\text { Young's } \\
\text { Modulus } \\
E / \mathrm{GP}_{\mathrm{a}}\end{array}$ & $\begin{array}{c}\text { Elongation } \\
\text { percentage } \\
\delta /(\%)\end{array}$ \\
\hline $\mathrm{SiC}_{\mathrm{w}} / 2024$ & 15 & 2.76 & 498 & 90.0 & 2.55 \\
$\mathrm{SiC}_{\mathrm{w}} / 2024$ & 20 & 2.80 & 500 & 97.0 & 2.5 \\
$\mathrm{SiC}_{\mathrm{w}} / 2024$ & 36 & 2.80 & 496 & 89.9 & 2.45 \\
\hline
\end{tabular}

\section{Experimental methods and detection instruments}

The experiments were done by cutting a discoid precision sample into $15 \mathrm{~mm} \times 15 \mathrm{~mm}$ (length $\times$ width) little slices with Model U250 wire-EDM precision machine. The power of machine was the wire-EDM high frequency pulse. The speed of moving wire was $0.1 \mathrm{~m} / \mathrm{s}$. The electrode had been made of $0.15 \mathrm{~mm}$-diameter molybdenum wire, which was connected to the negative pole of the power source, while the working component was geared into the positive pole of the power source. For other parameters, please refer to Table 2. The underlined figures in the table 2 were the benchmark parameters.

Table 2 Experimental parameters

\begin{tabular}{ccc}
\hline $\begin{array}{c}\text { Pulse width } \\
(\mu \mathrm{s})\end{array}$ & $\begin{array}{c}\text { Pulse interval } \\
(\mu \mathrm{s})\end{array}$ & Working fluid \\
\hline $10 、 \underline{19} 、 27 、 65 、 78,100$ & $19 、 43 、 \underline{60} 、 74 、 100$ & Deionized water、 $\underline{\text { Kerosene }}$ \\
\hline
\end{tabular}

The samples of wire electrical discharge machining were received under the conditions of testing listed in Table 3 and then they were sorted. It would be necessary to spray the gold film on the machined surface of sample before the detections of SEM. The SEM images would be measured with Model KYKY2800B scan electron microscope and the roughness value by Model Talysurf_6 roughometer.

Table 3 Conditions of testing

\begin{tabular}{cccc}
\hline $\begin{array}{c}\text { Test } \\
\text { No. }\end{array}$ & $\begin{array}{c}\text { Pulse width } \\
(\mu \mathrm{s})\end{array}$ & $\begin{array}{c}\text { Pulse interval } \\
(\mu \mathrm{s})\end{array}$ & $\begin{array}{c}\text { Working } \\
\text { fluid }\end{array}$ \\
\hline $1 \#$ & 10 & 60 & kerosene \\
$2 \#$ & 27 & 60 & kerosene \\
$3 \#$ & 65 & 60 & kerosene \\
$4 \#$ & 78 & 60 & kerosene \\
$5 \#$ & 100 & 60 & kerosene \\
$6 \#$ & 19 & 19 & kerosene \\
$7 \#$ & 19 & 43 & kerosene \\
$8 \#$ & 19 & 74 & kerosene \\
$9 \#$ & 19 & 100 & kerosene \\
$10 \#$ & 19 & 60 & kerosene \\
$11 \#$ & 19 & 60 & Deionized water \\
\hline
\end{tabular}

\section{Analysis and Discussion of the Experimental Results}

\section{The microstructures of machined surface}

As shown in Fig.1, which are the SEM images of microstructures of machined surface by electrical discharge machining. 

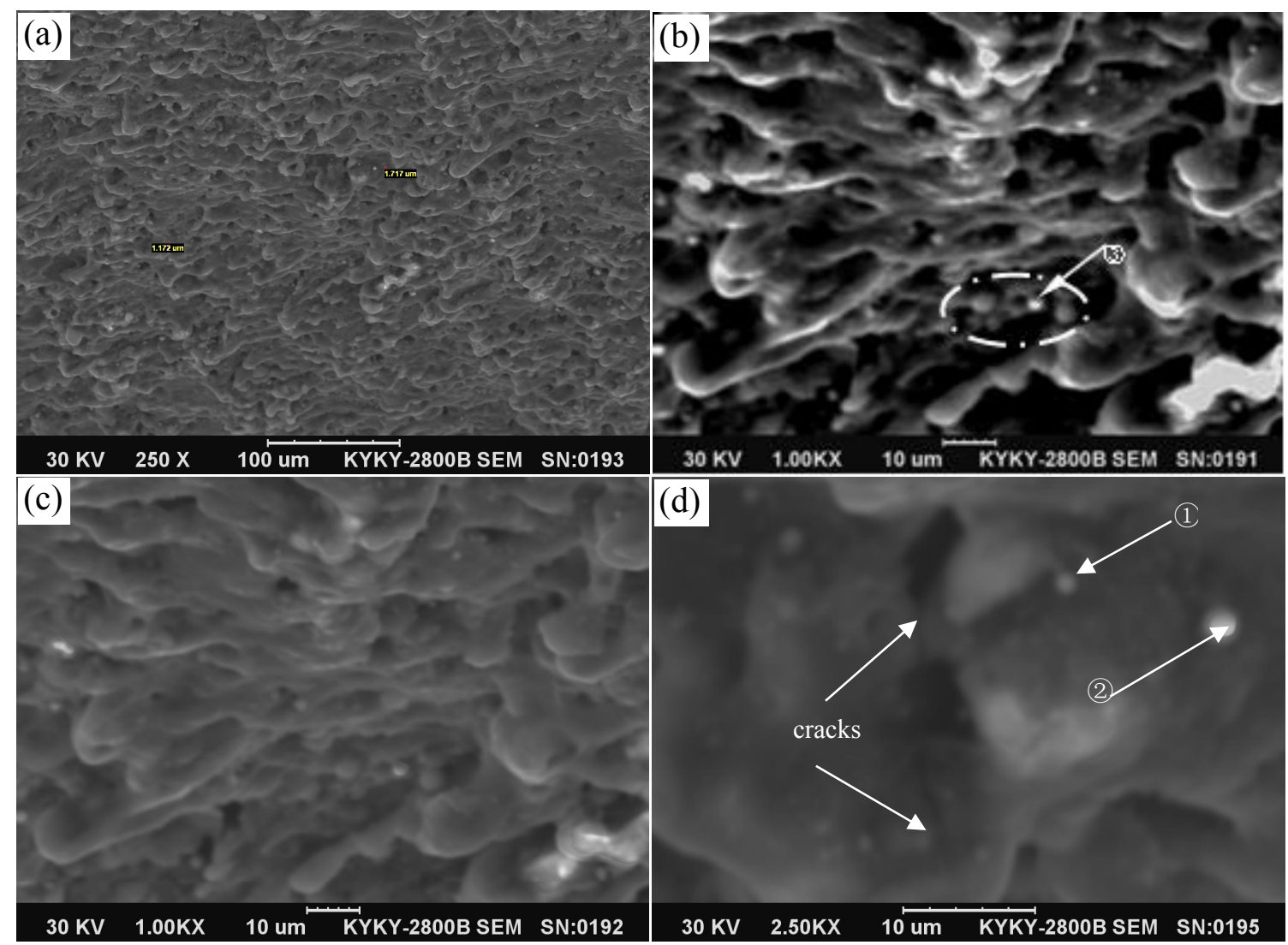

Fig.1 Microstructures of machined surface of samples

According to Fig.1, the processes of electrical discharge machining between $\mathrm{SiC}_{\mathrm{w}}$ reinforced aluminium composite and aluminium matrix alloy have both similarities and discrepancies. Summarily, there would be significant differences in the microprocess of discharging so that the microstructures of machined surface both are also different. From Fig.1, it can be seen that the microstructures of machined surface of $\mathrm{SiC}_{\mathrm{w}}$ aluminium matrix composite are very complicated. The microstructures could be allocated into the several kinds after the summary.

Microstructure A: pits

Based on Fig.1, the pits have the following possible sources:

(1)The instantaneous high temperature generated from discharging results in eroding the aluminum matrix material into a visible pit, which has a relatively large size and irregular shape, most of which present semilunar appearance.

(2) Under the impact of high temperature and thermal stress, the whisker wrapped by aluminium matrix is pulled out as the aluminium matrix is ejected, simultaneously. The pit shows a regular round shape, and the diameter is generally larger than $\mathrm{SiC}$ whisker (as shown in Fig.1, the diameter of the pit is $1.172 \mu \mathrm{m}$ and $1.717 \mu \mathrm{m}$, respectively), which has a shadow inside the hole due to the depth.

(3)The molten droplets splash when ejected at the high temperature, most of which are pulled out into working fluid, but there is still a part of the molten droplets to adhere to the machined surface after cooling. The "cofferdams" has a pit form that has a large size and irregular shape.

\section{Microstructure B: flanges or protuberances}

According to Fig.1, the generation of flange or protuberance has the following sources:

(1)In the single discharging area, the aluminium matrix, which has not been ejected or washed out by working fluid in the melt position, will generate flanges or protuberances surrounding a hole after cooling. As shown in Fig.1(b) and Fig.1(c), it would be possible to capture the pictures, where the dark areas are pits and bright areas are flanges or protuberances, after adjusting the focus of scan electron microscope(SEM).

(2)The molten droplets ejected by high temperature, some parts of which will cool and cohere with the machined surface again to form flanges or protuberances. 
(3) Since the difference of melting points that $\mathrm{SiC}_{\mathrm{w}}$ whisker and aluminium matrix hold, where the melting point of aluminium matrix is lower than that of $\mathrm{SiC}_{\mathrm{w}}$ whisker, there would be growing molten droplets generated from aluminium matrix fall into working fluid when processing. The exposure of $\mathrm{SiC}_{\mathrm{w}}$ whiskers could form protuberances on the machined surface.

\section{Microstructure C: spherical molten droplets of aluminium matrix}

The aluminium matrix is melted when machining, despite the major parts of them are pulled into working fluid, but there is still a tiny part of them to be adhered to the machined surface after cooling. As shown in Fig. 1(b), the marked oval part is the formation of molten droplets, which also has broken $\mathrm{SiC}_{\mathrm{w}}$ whisker in it.

\section{Microstructure D: cracks}

When employing electrical discharge machining, the surface is affected by the instantaneous high temperature, while the subsurface do not impact by the heat in time as the processing ceases immediately. Therefore, there is a high temperature gradient from the surface to the subsurface, which generates the heat stress as the experimental components become cooling. The tensile stress and pressure stress are produced in the surface and subsurface, respectively. As shown in Fig.1(d), a group of micro-flaws including cracks could be created on the machined surface when the residual stress reaches the limit of $\mathrm{SiC}_{\mathrm{w}}$ reinforced aluminium matrix composite.

Moreover, as indicated in Fig. 1 that there is a dark film on the pit and protuberance, which is the carbide film. Since the working fluid contains kerosene hydrocarbon, which has a high possibility to gather a large quantity of dissociative carbon particle on the machined surface. As shown in Fig.2, which is the figure $s$ of chemical element energy spectrum of machined surface, the element aluminium and the element silicon are the two kinds of main elements.

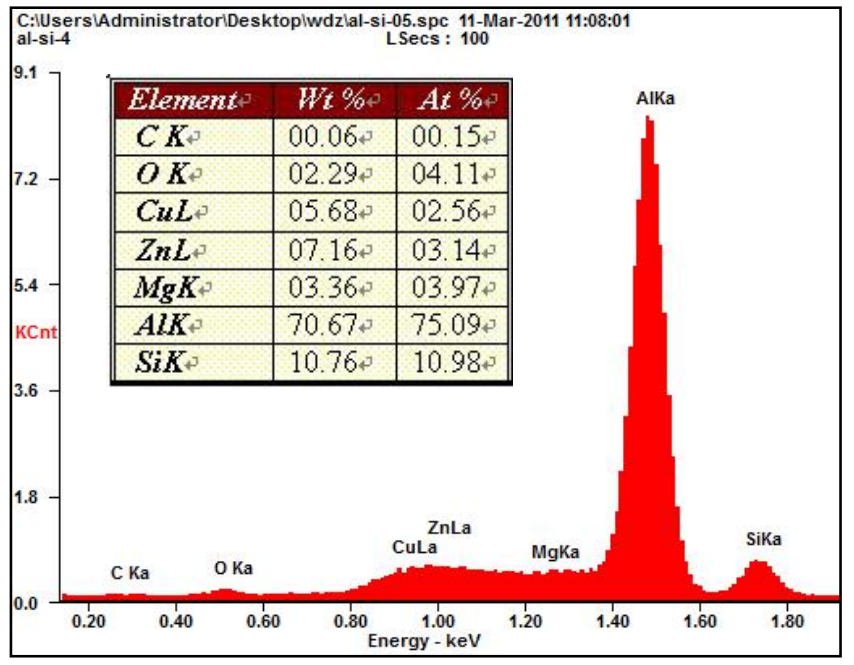

Fig.2 Chemical element energy spectrum of machined surface

\section{The broken mode of $\mathrm{SiC}_{\mathrm{w}}$ whiskers and existing features on the machined surface}

Since the melting point of $\mathrm{SiC}_{\mathrm{w}}$ whisker is approximately $2350^{\circ} \mathrm{C}$, while the temperature in electric discharging processing could produce a remarkable heat that is more than twice of $\mathrm{SiC}_{\mathrm{w}}$ whisker melting point $\left(5000^{\circ} \mathrm{C}\right)$, the melted $\mathrm{SiC}_{\mathrm{w}}$ whiskers could be either pulled out along with molten droplets of aluminium matrix and ejected to the working fluid; or attached to the remaining aluminium matrix. Therefore, the broken ways and existing mode of $\mathrm{SiC}_{\mathrm{w}}$ whiskers could be concluded in the several following modes:

(1)Mode melted: With the thermal stress in electric discharging processing, $\mathrm{SiC}_{\mathrm{w}}$ whiskers are just melted under high temperature along their interface, some segments of which are ejected to the working fluid along with melted aluminium matrix droplets; the rest segments are kept on the machined surface of testing components. The broken $\mathrm{SiC}_{\mathrm{w}}$ whiskers are the sources of convex points after cooling along with aluminium matrix. According to the white bright pints in Fig.1, the whiskers mentioned above are perpendicular to the surface of working components. 
(2)Mode pulled out: Along with the melted aluminium matrix ejected into the working fluid, the $\mathrm{SiC}_{\mathrm{w}}$ whiskers wrapped into the aluminium matrix droplets will be pulled out from their interface and to form some pits on the machined surface (as shown in Figure 1).

(3)Mode adhered again: $\mathrm{The} \mathrm{SiC}_{\mathrm{w}}$ whiskers wrapped into the droplets of the melted aluminium matrix will be embedded into the machined surface after the droplets cool down. The whisker breakages formed in the bonding again mode are distributed on the machined surface and their orientations are random and irregular.

\section{The impacts of processing speed and roughness value in electrical parameters}

\section{The effect pulse width on processing speed and roughness value}

As shown in Figure 3, it is the influence curve of pulse width for processing speed and roughness value. The data of the curves drawn with the Origin software come from the experimental measuring values of the specimen numbers $1 \# \sim 5 \#$ and 10\#. Based on the difference of the curve slope, the curves can be divided into three symbolic stages: low, medium and high pulse width stage, as shown in Figure 3 . In the process of low pulse width $(0 \mu \mathrm{s} \sim 30 \mu \mathrm{s})$ and high pulse width $(65 \mu \mathrm{s} \sim 100 \mu \mathrm{s})$, the slope of processing speed will slow increase with the increase of pulse width. However, the slope of processing speed will quickly increase with the increase of pulse width within the stage of medium pulse width $(30 \mu \mathrm{s} \sim 65 \mu \mathrm{s})$. In other words, the curve of this stage is becoming steeper than it of other stage. Moreover, the roughness values of three stages will linearly increase (the slope close to 1).

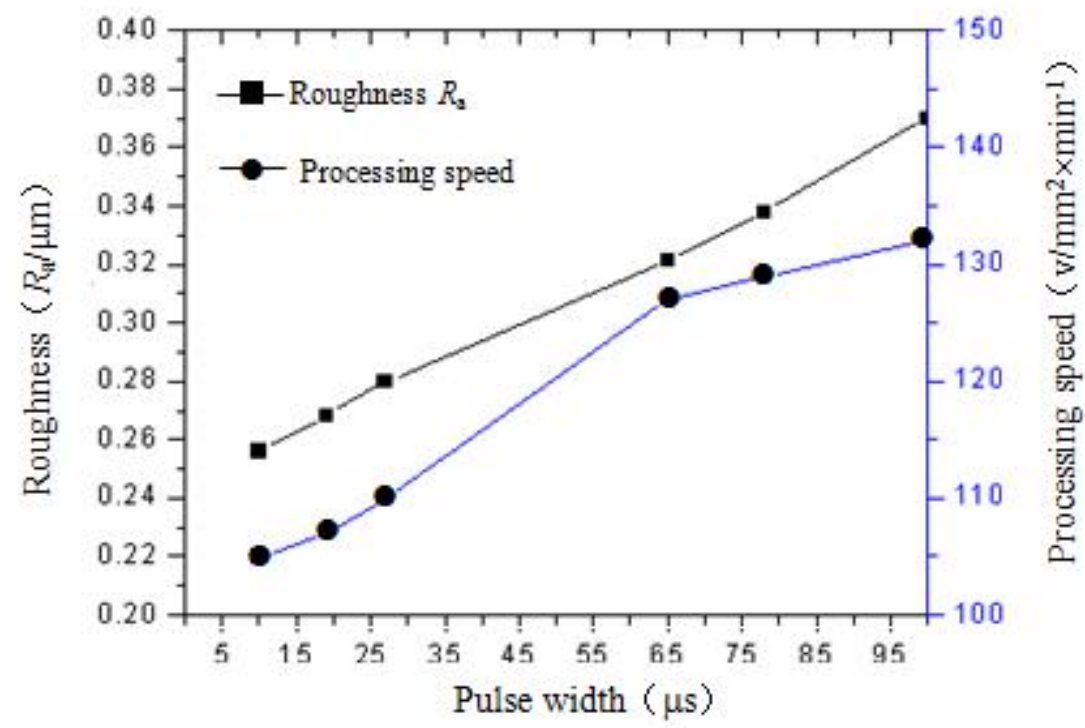

Fig.3 Influence curves of pulse width on processing speed and roughness value

The quantity of eroding in unit time would increase as a single impulse width broaden, which would exacerbate the power in the electric discharging channel.

Low impulse width stage $(0 \mu \mathrm{s} \sim 30 \mu \mathrm{s})$ : Since the impulse width and the power withhold are limited, the interface of eroding interacted in the aluminium matrix material, which would result in a major of $\mathrm{SiC}_{\mathrm{w}}$ whiskers are pulled out and adhered again. Thus, would set the obstacles to reduce the processing speed. In the meantime, the lower impulse width contributes to fewer derivatives in interelectrode corrosion, which could be transported smoothly by working fluid. That is why the increase in processing speed is restrained.

Medium impulse width stage $(30 \mu \mathrm{s} \sim 65 \mu \mathrm{s})$ : The power in the electrical discharging channel is getting immensurable, the most of $\mathrm{SiC}_{\mathrm{w}}$ whiskers are automatically melted when the impulse width reach a limit (threshold value). The production is carried out normally even though the derivatives of eroding in interelectrode are too many to ignore. That is why the processing speed boosted in a short period and the slope is steep compared to other stages.

High impulse width stage ( $65 \mu \mathrm{s} \sim 100 \mu \mathrm{s})$ : As the impulse width continuing increase, although the $\mathrm{SiC}_{\mathrm{w}}$ whiskers could be melted automatically as mentioned before, the derivatives have come to a 
large quantity, which are sufficient to impede the electric discharging processing when the producing speed has exceeded the flow speed of working fluid. That is why the increase in processing speed has declined.

Based on Figure 1(b) and 1 (c), the greater the power in the electrical discharging channel, the larger the radius of a single eroding pit and the deeper of its depth; the higher the edges of flanges, the higher the roughness value they hold. Figure 3 has provided us with an optimal impulse width for precision machining, which ranges from $19 \mu \mathrm{s}$ to $27 \mu \mathrm{s}$, for the roughness value rockets up if the impulse width reaches $27 \mu \mathrm{s}$. On the other hand, $65 \mu$ s would be fit for rough machining.

The impact of processing speed and roughness value in pulse interval

The impact of roughness is insignificant, however, as for wire-electrode processing speed, the influence is essential in pulse intervals. As shown in Figure 4, the curve is the results collected from Origin Software after inputting Serial Number 6\# 10\# data.

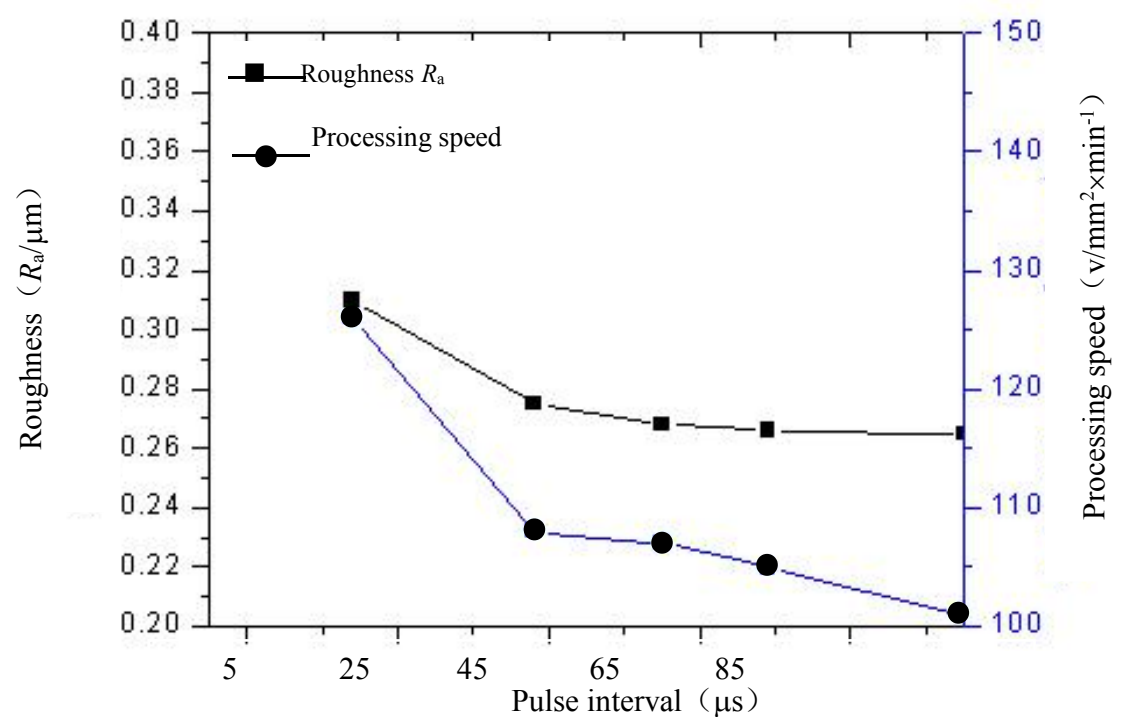

Fig.4 Influence curves of pulse interval for processing speed and roughness values

As shown in Figure 4, the wider the pulse interval is, the slower the processing speed is. And the fluctuation of roughness value is also constrained. The obvious processing speed reduction will happen within the pulse interval remaining in $19 \mu \mathrm{s}$ to $43 \mu \mathrm{s}$, there is a nearly flat decrease of processing speed mitigation when the pulse interval surpasses $43 \mu \mathrm{s}$. In addition, there is a slight drop of roughness value within the pulse interval $19 \mu \mathrm{s} \sim 60 \mu \mathrm{s}$ and would remain constant after the pulse interval $60 \mu \mathrm{s}$. The frequency of pulse has been mitigated, which means the numbers of electrical discharging in unit time has been minimized as the pulse interval will increase; the quantity of eroding in unit time has dropped, which triggers a reduction of processing speed.

Analysis shows that the frequency of EDM will decrease in some unit time with in crease of pulse interval so that the removal rate or the processing speed of EDM decreases. The wider the pulse interval is, the completer the deionization is done. Moreover, the insulation of the medium between two electrodes will keep the good performance. Because of the stable process of EDM, the machined surface will not appear larger concaves, flanges or protuberance so that the roughness values of machined surface will maintain the stability.

\section{Conclusions}

It is feasible to apply wire-EDM in machining $\mathrm{SiC}_{\mathrm{w}} / 2024$ aluminium matrix composite, which could obtain a superior quality of machined surface. 
Compared to general alloys, $\mathrm{SiC}_{\mathrm{w}}$ reinforced aluminium matrix composite has a more complicated microstructure, which resembles 'birds nest', constructed main with pits, flanges, protuberances, aluminium droplets adhered again and cracks etc.

The main parameters in electrical discharge machining, the processing speed and roughness value, are extremely affected by pulse width and pulse interval.

\section{Acknowledgement}

This work was financially supported by the Natural Science Foundation of Fujian of China (No. 2015J01215)

\section{References}

[1] D. Z. Wang, P. F. Feng, Q. L. Zhao. Journal of Astronautics, Vol.27(6),p.1341-1346(in Chinese).

[2] B. Mohan, A. Rajadurai, G. Satyanarayanak. Journal of materials processing technology, 2004, (153-154),p.978-985.

[3] B. Mohan, A. Rajadurai, G. Satyanarayanak. Journal of materials processing technology, 2002, (124),p.297-304.

[4] C. K. Srinivasa, A. R. Vinod. International Journal of Materials and Product Technology, 2012, (43),p.68-83.

[5] D. Satishkumar, M. Kanthabu. International Journal of Advanced Manufacturing Technology, 2011, (56),p.975-986.

[6] J. G. Li, J. Wang. Advanced Material Research, 2012, (347-353),p.1088-1092.

[7] C. Senthilkumar, G. Ganesan. Materials Science and Technology,2010,(26),p. 289-296.

[8] W. S. Yang, G. Q. Chen, P. Wu. Acta Metallurgica Sinica (English Letters), 2017, (30),p. 447-455.

[9] N. G. Patil, P. K. Brahmankar. 18th CIRP Conference on Electro Physical and Chemical Machining, ISEM 2016, Procedia CIRP, 2016,(42), p.286-291.

[10]D. K. Kasdekar, V. Parashar, P. Soni. Composite Materials and Material Engineering (Materials Science Forum), 2016, (860), p.61-64.

[11]H. S. Pali, N. Kumar, K. Singh. SAE 2016 World Congress and Exhibition, SAE Technical Papers, 2016-April 5. 